(REVIEW ARTICLE)

\title{
A review on cubosome: The novel drug delivery system
}

\author{
Sadhu Venkateswara Rao ${ }^{1 *}$, Beram Naga Sravya ${ }^{1}$ and Kantamneni Padmalatha ${ }^{2}$ \\ ${ }^{1}$ Department of Pharmaceutics, Vijaya Institute of Pharmaceutical Sciences for Women, Enikepadu, Vijayawada-521108, \\ India. \\ ${ }^{2}$ Department of Pharmacology, Vijaya Institute of Pharmaceutical Sciences for Women, Enikepadu, Vijayawada-521108, \\ India.
}

Publication history: Received on 21 August 2018; revised on 28 September 2018; accepted on 05 October 2018

Article DOI: https://doi.org/10.30574/gscbps.2018.5.1.0089

\begin{abstract}
Cubosomes are nanoparticles in structure which is mainly made of certain amphiphilic lipids in definite proportion, known as bicontinuous cubic phase liquid crystals. Hydrating a surfactant or polar lipid that forms cubic phase and then dispersing a solid like phase into smaller particles usually forms a cubosomes. They perform solid like rheology with unique properties of practical interest. They are thermodynamically stable and they have carvenous (honeycomb) structures which are tightly packed twisted into three dimensional bilayers. This type of complex structure allows them to have greater drug loading ability. Cubosomes have ability to encapsulate the hydrophobic, hydrophilic, amphiphilic substances. Cubosomes can increase the solubility of poorly soluble drug. Cubosome dispersions are bioadhesive and biocompatible. Because of their properties, cubosome are versatile systems, administrable by different ways such as orally, percutaneously and parenterally. Cubosome structure by means of electron microscopy, light scattering, $\mathrm{x}$-ray and NMR, nevertheless few researchers has been studying the potential of cubosome as delivery systems.
\end{abstract}

Keywords: Cubosomes; Nanoparticles; Bicontinuous; Honeycomb

\section{Introduction}

Cubosomes are discrete, sub-micron, nanostructured particles of the bicontinuous cubic liquid crystalline phase. The term Cubosomes was coined by Larsson, which reflects the cubic molecular crystallography and similarity to liposomes. These are nanoparticles which are self-assembled liquid crystalline particles of certain surfactants with proper ratio of water with microstructure. Cubosomes are nanoparticles but instead of the solid particles usually encountered, these are self-assembled liquid crystalline particles with a solid like rheology that provides unique properties of practical interest [1]. Most probably cubosomes are composed of polymers, lipids and surfactants with polar and non-polar components hence said as amphiphilic. The amphiphilic molecules are driven by the hydrophobic effect into polar solvent to impulsively identify and assemble into a liquid crystal of nanometer scale. Thus cubosomes are bicontinuous cubic liquid phase enclosing two separate regions of water divided by surfactant controlled bilayers. Further these are similar to liquid crystalline substance with cubic crystallographic symmetry and are optically isotropic, viscous and solid too. The cubic phase can fracture and form colloidally and thermodynamically stable particulate dispersions. Cubosomes have great importance in nanodrug formulations and are formations of bicontinuous cubic liquid crystalline phase by hydrating mixture of monoolein and poloxamer 407 [2]. Dots Square shaped, slightly spherical of $10-500 \mathrm{~nm}$ in diameter. In cubosomes active chemical constituent molecules are anchored through chemical bonds to the polar head of the phospholipids. The polymer and the individual drug compound form a 1:1 or 2:1 complex depending on the substance.

\footnotetext{
${ }^{*}$ Corresponding author

E-mail address: venkateshsadhu@gmail.com
} 
Despite the early recognization (in 1980) large scale manufacture of cubosomes was difficult due to their complex phase behavior and viscous properties. Certain surfactants spontaneously form cubic phases when mixed with water at certain concentration [3]. Effort to develop scalable processes to produce cubosomes in large scale is under development. A few anticancer drugs have been successfully encapsulated in cubosomes and characterized.

\subsection{Advantages}

1. It is economic.

2. It is non-toxic and biocompatible.

3. Method of preparation is simple.

4. It has excellent bio adhesive properties.

5. It has skin permeation enhancement.

6. For longer time they are thermodynamically stable.

7. Capability of encapsulating hydrophilic, hydrophobic and amphiphilic substances.

8. Targeted release and controlled release of bioactive agents.

9. Due to high internal surface area \& cubic crystalline structures there is high drug loading.

\subsection{Disadvantages}

1. Due to presence of large amounts of water inside cubosomes there is low entrapment of water soluble drugs $[4]$.

2. Because of the high viscosity the large scale production is sometimes difficult.

3. Large scale production is difficult for sometimes because of high viscosity [5].

\section{Structure of cubosome}

The basic structure of cubosomes includes honeycombed structures separating the two internal aqueous channels along with large interfacial area. Cubosomes are nanoparticles, more accurately nanostructure particles of liquid crystalline phases with cubic crystallographic symmetry formed by the self-assembly of amphiphilic or surfactant like molecules. The cubic phases possess a very high solid like viscosity, which is a unique property because of their intriguing bicontinuous structures which enclose two distinct regions of water separated by a controlled bilayer of surfactant application [6]. Amphiphilic molecules form bicontinuous water and oil channels, where bicontinuous refers to two distinct (continuous, but non-intersecting) hydrophilic regions separated by the bilayer. The interconnectedness of the structure results in a clear viscous gel similar in appearance and rheology to cross-linked polymer hydrogels.

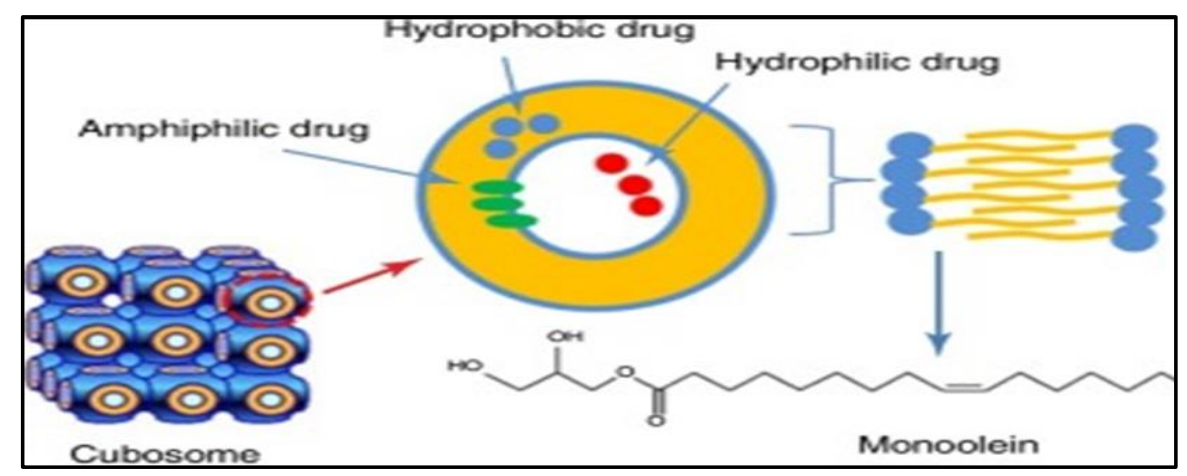

Figure 1 Structure of cubosome separating two internal aqueous channels along with large interfacial area

\section{Materials used in cubosome formation}

Bicontinuous cubic phases are found in natural lipids, cationic and non-ionic surfactants and polymer systems [7]. The lipid most widely used to construct bicontinuous cubic phases is the monoglyceride monoolein, monoglycerides spontaneously form bicontinuous cubic phases upon the addition of water, are relatively insoluble and are resistant to changes in temperature. The main precursor of cubosome formation is monoolein. Monoolein or glycerylmonooleate is a mixture of the glycerides of oleic acid and other fatty acids, consisting mainly the monooleate [8]. Monoolein may be obtained in two forms, a mixed glyceride form or as distilled monoolein; the distilled monoolein is preferred for pharmaceutical applications because of its high purity. Monoolein occurs as a waxy yellow paste with a characteristic odour. It swells in water, giving rise to several lyotropic liquid crystalline structures. Monoolein is a nontoxic, 
biodegradable and biocompatible material classified as GRAS (generally recognized as safe) and it is included in the FDA inactive ingredients guide and in nonparenteral medicines licensed in the United Kingdom. Monoolein show the mesomorphic phase, important in making more comprehensible the potential pharmaceutical application of the lipid.

In general monoglycerides exhibit different phase behaviours when they exposed to water. Surfactants, which are used in the production of cubosomes, are poloxamer 407 in a concentration range between $0 \%$ and $20 \% \mathrm{w} / \mathrm{w}$ with respect to the disperse phase. The concentration of the monoglyceride/surfactant mixture generally takes between $2.5 \%$ and $10 \% \mathrm{w} / \mathrm{w}$ with respect to the total weight of the dispersion. Polyvinyl alcohol used in alternative to poloxamer as stabilizing agent in the dispersion.

\section{Method of preparation}

Cubosomes can be manufactured by two defferent techniques as shown in figure 2 .

1. TOP DOWN TECHNIQUE

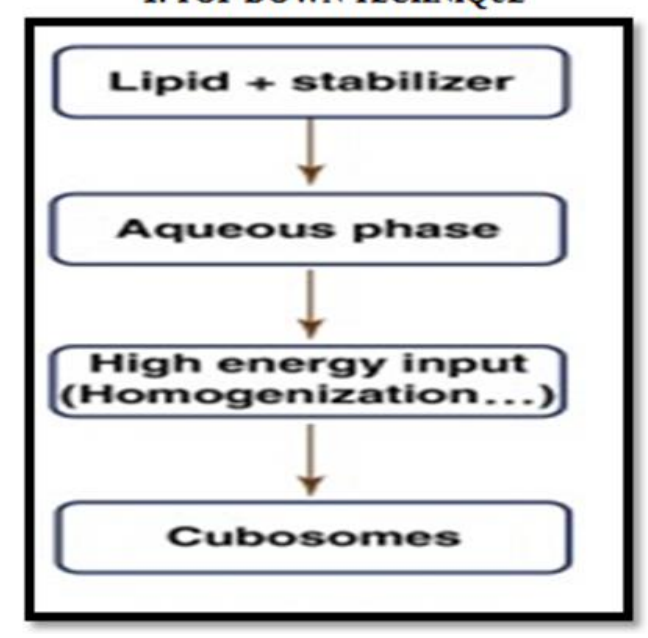

Figure 2 Manufacturing techniques of cubosomes

\section{BOTTOM UP TECHNIQUE}

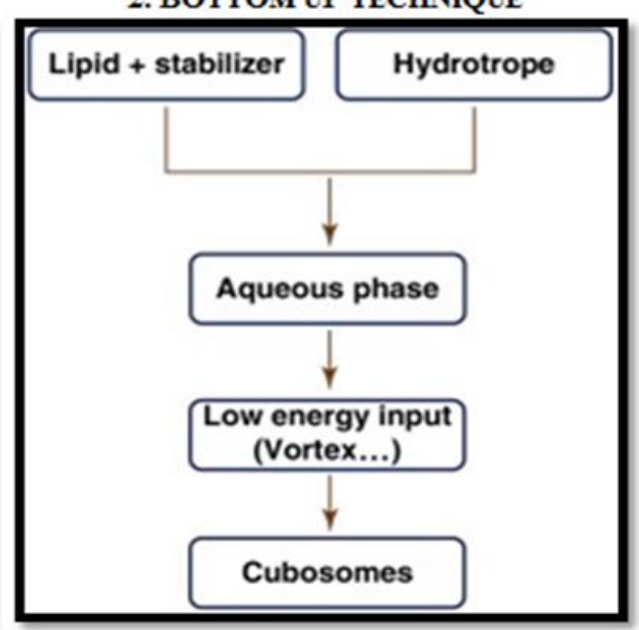

The cubosome dispersion carried out by two methods

\subsection{Fabrication method}

GM0/p407 cubic gel GMO 5\% and P407 1.0\% were melted at $60^{\circ} \mathrm{C}$ in hot water bath and add the required amount of drug and stir continuously till dissolve. Deionized water is added drop by drop and vortex is set to the homogenisation. It kept upto $48 \mathrm{hrs}$ at room temperature the optically isotropic cubic gel are formed and disturbed by mechanical stirring crude dispersion was subsequently fragmented by sonicater probe having the energy $200 \mathrm{~W}$ under cool temperature at the $20^{\circ} \mathrm{C}$ in water bath for the $20 \mathrm{~min}$ [9].

\subsection{Emulsification method}

In this method the GMO and P407 are put in to the water and it followed the ultrasonication the 5\% GMO and1\% P407and 5\% ethanol in 89\% water are taken GMO and $\mathrm{P} 407$ are melted at the $60^{\circ} \mathrm{C}$ and mixed the ethanolic solution was added to the melting. The resultant mixture are added drop wise to deionized water preheated at the $70^{\circ} \mathrm{C}$, it ultrasonicated at maximum power $130 \mathrm{~kW}$ for $50 \mathrm{~min}$ at the same temperature the disperse solution are kept in to the ambient temperature and protected from light [10].

\section{Evaluation of cubosomes}

\subsection{Visual inspection}

The cubosomes are visually assessed for optical appearance (e.g colour, turbidity, homogeneity, presence of macroscopic particles). 


\subsection{Shape of the cubosome}

Transmission electron microscopy can be used to view the shape of the cubosomes.

\subsection{Particle size distribution}

Particle size distributions of cubosomes are mainly determined by dynamic laser light scattering using Zeta sizer (Photon correlation spectroscopy). The sample diluted with a suitable solvent is adjusted to light scattering intensity of about $300 \mathrm{~Hz}$ and measured at $25^{\circ} \mathrm{C}$ in triplicate [11]. The data can be collected and generally shown by using average volume weight size. The zeta potential and polydispersity index can also be recorded.

\subsection{Zeta potential}

The magnitude of zeta potential indicates the degree of electronic repulsion between adjust, similarly charge particle. Zeta potential is key indicator of the stability of formulation.

\subsection{Entrapment efficiency}

The entrapment efficiency of cubosomes can be determined using ultra filtration techniques [10]. In the later technique, unentrapped drug concentration is determined, which is subtracted from the total drug added. The amount of drug is analyzed by using spectrophotometer.

\subsection{Measurement of drug release}

Drug release from cubosomes can be done by pressure ultrafiltration method [10]. It is based on that proposed by Magenheim et al. using an Amicon pressure ultrafiltration cell fitted with a Millipore membrane at ambient temperature $(22 \pm 2)^{\circ} \mathrm{C}$.

\subsection{Stability studies}

The physical stability can be studied by investigation of organoleptic and morphological aspects as a function of time. Particle size distribution and drug content can be assessed at different time intervals can also be used to evaluate the possible variations by time [12].

\section{Applications}

\subsection{In cancer therapy}

Recently some anticancer drugs have been successfully encapsulated in cubosomes and characterized physicochemical properties [13]. The unique structure of this promising nano carrier suggests its application in melanoma therapy. In order to specifically target nano medicines to tumours, different approaches have been envisaged, with passive and active targeting of cancer cells having been shown to be valid approaches in preclinical and clinical studies.

\subsection{Oral drug delivery}

Cubosomes direct the varied challenges in oral delivery of numerous compounds including poor aqueous solubility, poor absorption, and large molecular size. In an application large proteins have been encapsulated for local activity in the gastrointestinal tract [14]. Cubosomes technology provides drug release at different absorption sites, for example in the upper or lower intestine, which is important for the drugs that have narrow absorption window.

\subsection{Intravenous drug delivery systems}

Lipid nanoparticles comprising interior liquid crystal structures of curved lipid membranes are used to solubilize encapsulate and deliver medications to disease areas within the body [15]. Compare to emulsions and liposomes the cubosomes nanoparticle shows increased payloads of peptides, proteins and many insoluble small molecules, and are ideal carriers for injection.

\subsection{Topical drug delivery systems}

Cubosomes are more bioadhesive in nature, so that they can conveniently use in topical and mucosal delivery of different drugs. Topical delivery systems are based on the exploitation of unique properties of liquid crystal and liquid crystal nanoparticle technologies. Topical drug delivery systems are unique in situ forming bioadhesive liquid crystal systems facilitate controlled and effective drug delivery to mucosal surfaces like buccal, ophthalmic and vagina. 


\subsection{Drug delivery vehicle}

Drug delivery vehicle is a common application for such new materials. The research in association with cosmetic companies like L'Oreal and Nivea are trying for the use of cubosome particles as oil-in-water emulsion stabilizers and pollutant absorbents in cosmetics [16].

\subsection{Controlled or sustained release behaviour}

Number of drugs with different physicochemical properties has been incorporated in cubosomes and their sustained drug release behaviour was also studied. Sustained behavior of cubosomes was because of cubosome remnant particles. Monoglyceride based cubosome can be proposed for topical use, such as for perctuneous or mucosal application.

\subsection{In treatment of viral diseases}

Because of the microbicidal properties of monoglycerieds, could be used to design intravaginal treatment of sexually transmitted diseases caused by viruses (e.g. HSV, HIV) or by bacteria like Chlamydia trachomatis and Neisseria genorrticae [17].

\section{Future prospects}

The cubosome nanoparticles hold promise in the field of drug delivery and sustained drug release, but further optimization is still required, depending on the route of administration, frequency of dosing and the mode of drug release, before such nanocarriers can truly realize their therapeutic potential in many diseases [18]. They are also attractive nanovehicles for loading and delivery of proteins and peptides but the reported studies are still on a fundamental level [19] and different aspects in terms of structural and morphological characterstics of these soft nanocarriers, loading capacity of bio macromolecules and their release should be addressed. Future development of cubosome based intravenous nanomedicines should address blood compatability at early stages of formulation development. Further, little information is also still available on their stability in biological fluids and biological factors controlling drug release from cubosomes, structural transformation upon contact with biological fluids such as plasma, interactions with cell membranes, and infusion-related reactions to name a few [20]. The application of cubosomes for intravenous drug delivery is an ambitious one; however, these nanocarriers may find accelerated applications for oral, ocular and topical delivery of poorly water soluble drugs, there by offering an alternative, yet, a cost effective opportunity in formulation science.

\section{Conclusion}

Cubosomes are nanoparticles but instead of the solid particles, cubosomes are self-assembled liquid crystalline particles, they have ability to incorporate many hydrophilic and lipophilic drugs and shows sustained and targeted drug delivery. Two methods such as top down and bottom up approaches could be easily employed to produce cubosomes either by ultrasonication techniques or high pressure homogenization. Cubosomes are applicable to wide range of drug candidates, proteins, immune substances and also to cosmetics. Due to the potential site specificity, the cubosomal preparations may be widely employed as targeted drug delivery systems for ophthalmic, diabetic and also for anticancer therapy. The cubosome technology is relatively new with high output and would have wide scope of research in developing new formulations with commercial and industrial viability.

\section{Compliance with ethical standards}

\section{Acknowledgments}

The presenting authors are thankful to principal, Vijaya institute of pharmaceutical sciences for women, Vijayawada for their valuable support in carrying out this work.

\section{Disclosure of conflict of interest}

All authors declare that they have no conflict of interest.

\section{References}

[1] Almeida JD, Brand CM, Edwards DC and Heath TD (1975). Formation of virosomes from influenza subunits and liposomes. Lancet. 2, 899-901. 
[2] Spicer PT. (2004). Cubosomes bicontinuous cubic liquid crystalline nanostructured particles. The Procter and Gamble Company, West Chester, Ohio, USA.

[3] Rizwan SB, Dong YD, Boyd BJ, Rades T and Hook S. (2007). Characterisation of bicontinuous cubic liquid crystalline systems of phytantriol and water using cryo field emission scanning electron microscopy. Micron. 38, 478-485.

[4] Tilekar KB, Khade PH, Kakade S, Kotwal S and Patil R. (2014). Cubosomes a drug delivery system. International Journal of Chemical and Biochemical Science. 4, 812-824.

[5] Karami Z and Hamidi M. (2016). Cubosomes: Remarkable drug delivery potential. Drug Discovery Today. 21, 789-801.

[6] Urvi S, Dhiren D, Bhavin P, Patel U and Shah R. (2013). Overview of cubosomes: A Nanoparticle. In. J of Ph. and Integ. Life Sci., 1(5), 36-47.

[7] Stroem P and Anderson DM. (1992). The cubic phase region in the system didodecyl dimethyl ammonium bromide-water-styrene. Langmuir. 8(2), 691-709.

[8] Engström S, Larsson K and Lindman B. (1998). Controlled Release Bioact. Mater. 15, 105-106.

[9] Bhowmik D, Gopinath H, Kumar BP, Duraivel S and Kumar KS. (2012). Recent advances in novel topical drug delivery system. The Pharma Innovation. 1(9), 12.

[10] Thorat YS, Gonjari ID and Hosmani AH. (2011). Solubility enhancement techniques: a review on conventional and novel approaches. International journal of pharmaceutical sciences and research. 2(10), 2501.

[11] Bhosale RR, Osmani RA, Harkare BR and Ghodake PP. (2013). The Inimitable Nanoparticulate Drug Carriers. Scholars Academic Journal of Pharmacy. 2(6), 481-486.

[12] Saly S, Ehab RB and Sabry B. (2013). The design and evaluation of novel encapsulation technique for topical application of alpha lipoic acid. Journal of Advanced Pharmaceutical Research. 4(1), 13-22.

[13] Sagnella S and Drummond C. (2012). Drug delivery a nanomedicine approach. Australian Biochemist. (43), 5-7.

[14] Bei D, Meng J and Youan BC. (2010). Engineering nanomedicines for improved melanoma therapy progress and promises. Nanomedicine (London, England), 5(9), 1385-1399.

[15] Angelov B, Angelova A and Garamus VM. (2012). Earliest stage of the tetrahedral nanochannel formation in cubosome particles from unilamellar nanovesicles. Langmuir. 28(48), 16647-16655.

[16] Angelov B, Angelova A and Drechsler M. (2015). Identification of large channels in cationic PEGylated cubosome nanoparticles by synchrotron radiation SAXS and Cryo- TEM imaging. Soft Matter. 11(18), 3686-3692.

[17] Thadanki M, Kumari PS and Prabha KS. (2011). Overview of cubosomes: a nanoparticle. International Journal of Research in Pharmacy and Chemistry. 1(3), 535-541.

[18] Angelova A, Angelov B and Lesieur S. (2008). Dynamic control of nanofluidic channels in protein drug delivery vehicles. Journal of Drug Delivery Science and Technology. 18(1); 41-45.

[19] Angelova A, Angelov B and Drechsler M. (2013). Protein entrapment in PEGylated lipid nanoparticles. International Journal of Pharmac. 454(2), 625-632.

[20] Wibroe PP, Azmi ID, Nilsson C, Yaghmur A and Moghimi SM. (2015). Citrem modulates internal nanostructure of glyceryl monooleate dispersions and bypasses complement activation: towards development of safe tunable intravenous lipid nanocarriers. Nanomedicine. 11(8), 1909-1914.

\section{How to cite this article}

Sadhu VR, Beram NS and Kantamneni P. (2018). A review on cubosome: The novel drug delivery system. GSC Biological and Pharmaceutical Sciences, 5(1), 76-81. 